\title{
PHRAGMÈN-LINDELÖF THEOREMS FOR ELLIPTIC EQUATIONS IN THE PLANE
}

\author{
BY \\ J. K. ODDSON
}

1. Introduction. Theorems of Phragmèn-Lindelöf type and other related results for elliptic-parabolic equations have been given by numerous authors in recent years ([1]-[10], for example). Most of these results concern elliptic equations in a half-space although in [5] conical domains are considered under the additional assumption that the principal part of the elliptic operator approaches the Laplacian at infinity. More generally, results of a qualitative nature have been obtained in [17] without this assumption. Theorems of a different but related type involving growth conditions on the $L_{2}$ norm of a solution in general domains are given in [11].

In this paper we wish to consider a class of uniformly elliptic differential operators in two-dimensional domains which are contained within a half strip or a sector. In the case of the Laplacian the Phragmèn-Lindelöf theorem for such domains is easily obtained from the result for the half plane by conformal mapping; however for more general elliptic operators this technique is not available. As a result the Phragmèn-Lindelöf growth index (the critical rate of growth of solutions for which the theorem first fails) has been unknown up to now $\left({ }^{1}\right)$. Our purpose here is to establish this growth index for half strips and sectors, showing explicitly its dependence upon the constant of ellipticity and the size of the enclosing domain (width of the half strip or angle of the sector), and to verify that it is best possible for the class of operators which we consider. The appropriate Phragmèn-Lindelöf indices are defined in $\$ 2$ and our main results are contained in Theorems 3.3, 4.1, and 4.2.

We also include some immediate applications to extended maximum principles and uniqueness theorems.

2. Notations and basic hypotheses. Let $D$ be an open, connected subset of $E^{n}$ $(n \geqq 1)$ and denote by $\mathscr{L}_{\alpha}(D)$ the class of second order elliptic differential operators of the form

$$
L=\sum_{i j=1}^{n} a_{i j}(x) \frac{\partial^{2}}{\partial x_{i} \partial x_{j}}
$$

with coefficients defined in $D$ and satisfying there the normalization:

$$
\sum_{i=1}^{n} a_{i i}(x)=1 \quad \forall x \in D
$$

Received by the editors October 18, 1968 and, in revised form, May 15, 1969.

$\left.{ }^{1}\right)$ The existence of such an index for conical domains in $n \geqq 3$ dimensions is proven in [17]. Copyright (C) 1969, American Mathematical Society 
and the ellipticity condition:

$$
\sum_{i j=1}^{n} a_{i j}(x) \xi_{i} \xi_{j} \geqq \alpha \sum_{i=1}^{n} \xi_{i}^{2} \quad \forall x \in D
$$

for all real $n$ vectors $\left(\xi_{1}, \ldots, \xi_{n}\right)$ and some constant $\alpha$ in the range $0<\alpha \leqq 1 / n$.

Clearly any second order uniformly elliptic operator (linear, quasilinear, or nonlinear) belongs, after normalization, to some class $\mathscr{L}_{\alpha}$. In particular note that the class $\mathscr{L}_{1 / n}$ consists of the single operator $(1 / n) \Delta$ where $\Delta$ is the $n$-dimensional Laplacian. For quasilinear or nonlinear operators the ellipticity constant $\alpha$ will in general depend upon the particular solution function being considered.

In recent papers [12], [13] we have given a partial axiomatization of PhragmènLindelöf techniques, based upon the maximum principle and the assumption of the existence of suitable auxiliary comparison functions. For the special class of operators which we consider here a typical Phragmèn-Lindelöf result obtained in these papers may be stated as

TheOREM 2.1. Suppose that $\partial D=\Gamma \cup \Gamma_{1}$, where $\Gamma$ and $\Gamma_{1}$ are disjoint sets. Let $u(x)$ be a real valued function, twice differentiable in $D$, such that $L u(x) \leqq 0$ in $D$ for some $L \in \mathscr{L}_{\alpha}$ and $\lim \inf _{x \in D: x \rightarrow \Gamma_{1}} u(x) \geqq 0$. Suppose that there exists a nonnegative function $V(x)$, twice differentiable in $D$, such that $L V \leqq 0$ in $D$. Define $u^{-}(x)=$ $\max \{0,-u(x)\}$ and suppose that $u^{-}(x)=o\{V(x)\}$ as $x \rightarrow \Gamma, x \in D$. Then $u \geqq 0$ in $D$.

Here all topological concepts are understood to be with respect to $\overline{E^{n}}$, the one point compactification of $E^{n}$ so that if $D$ is unbounded in $E^{n}$ the boundary of $D$ will include the point at infinity.

The successful application of Theorem 2.1 is clearly reduced to the problem of constructing a suitable comparison function $V(x)$. In [13] we have indicated how this may be accomplished for the entire class $\mathscr{L}_{\alpha}$ with the aid of the maximizing equation for the class, previously introduced by Pucci [14], [15], provided this nonlinear equation can be solved. In a recent paper [16] such solutions have been obtained for strips and sectors in the plane $(n=2)$ and provide optimum comparison functions for the operators of the class $\mathscr{L}_{\alpha}$ in such domains. We devote the remainder of this section to a description of these functions. Note that for plane domains our fixed constant $\alpha$ is restricted to the range $0<\alpha \leqq \frac{1}{2}$.

We consider first the plane half strip $H(d)=\{(x, y):-d<x<d, y>0\}$ and define the Phragmèn-Lindelöf index

$$
\lambda(d ; \alpha)=\frac{1}{2 d}\left\{\frac{1}{\alpha(1-\alpha)} \tan ^{-1}(\alpha /(1-\alpha))^{1 / 2}-\frac{(1-2 \alpha)}{(\alpha(1-\alpha))^{1 / 2}}\right\}
$$

and the periodic function $C(t ; \alpha)$ in the parametric form

$$
\begin{aligned}
C(t ; \alpha) & =\frac{\cos \varphi}{1+(1-2 \alpha) \cos \varphi} \exp \left\{\frac{-(1-2 \alpha) \cos \varphi}{1+(1-2 \alpha) \cos \varphi}\right\} \\
t & =(4 \alpha(1-\alpha))^{1 / 2} \int_{0}^{\varphi} \frac{d \xi}{[1+(1-2 \alpha) \cos \xi]^{2}}
\end{aligned}
$$


The function

$$
V(x, y)=e^{\lambda y} C(\lambda x ; \alpha),
$$

positive in the half strip $H(d)$ and vanishing on its vertical sides, is the comparison function which we seek for $H(d)$. It has been obtained in [16] as a solution of the maximizing equation relative to the class $\mathscr{L}_{\alpha}$. It follows from the theory of extremal operators [15] that for every operator $L \in \mathscr{L}_{\alpha}$ we have

$$
L V(x, y) \leqq 0 \quad \forall(x, y) \in H(d) .
$$

Furthermore there exists an operator $L^{\prime} \in \mathscr{L}_{\alpha}$ such that $L^{\prime} V \equiv 0$ in $H(d)$.

We note that if $\alpha=\frac{1}{2}$, in which case the class $\mathscr{L}_{1 / 2}$ contains only the single operator $\left(\frac{1}{2}\right)\left\{\partial^{2} / \partial x^{2}+\partial^{2} / \partial y^{2}\right\}$, our comparison function reduces to the harmonic function

$$
V(x, y)=e^{\pi y / 2 d} \cos (\pi x / 2 d) .
$$

For the plane sector $S(\beta)=\{(x, y): r>0,|\theta|<\beta<\pi\}$ the construction of comparison functions is more involved. If $0<\alpha<\frac{1}{2}$ we define the constants $\zeta_{1}=$ $\cos ^{-1}(1-2 \alpha) \in(0, \pi / 2), \zeta_{2}=\pi-\zeta_{1}$ and the Phragmèn-Lindelöf indices

$$
\mu(\beta ; \alpha)=\frac{2(1-2 \alpha)}{\cos \zeta+(1-2 \alpha)}
$$

$$
=\frac{2(1-2 \alpha)}{\cosh \zeta+(1-2 \alpha)}
$$

and

$$
\begin{aligned}
\nu(\beta ; \alpha)=\frac{2(1-2 \alpha)}{\cos \zeta-(1-2 \alpha)} \quad \text { where } \quad & \zeta \in\left(0, \zeta_{1}\right) \text { satisfies } \\
& {[\zeta / \tan \zeta] \tan \zeta_{1}-\zeta_{1}=2 \beta \text { when } } \\
& 0<2 \beta<\min ^{m}\left\{2 \pi, \tan \zeta_{1}-\zeta_{1}\right\} \\
=\frac{2(1-2 \alpha)}{\cosh \zeta-(1-2 \alpha)} \quad \text { where } \quad \zeta \in[0, \infty) \text { satisfies } & \\
& {[\zeta / \tanh \zeta] \tan \zeta_{1}-\zeta_{1}=2 \beta \text { when } } \\
& \tan \zeta_{1}-\zeta_{1} \leqq 2 \beta<2 \pi
\end{aligned}
$$
where $\left\{\begin{array}{l}\zeta \in\left[\zeta_{1}, \zeta_{2}\right) \text { satisfies } \\ {[(\pi-\zeta) / \tan \zeta] \tan \zeta_{1}+\zeta_{1}=2 \beta} \\ \text { when } 0<2 \beta \leqq \pi \\ \zeta \in\left(0, \zeta_{1}\right) \text { satisfies } \\ {[\zeta / \tan \zeta] \tan \zeta_{1}+\zeta_{2}=2 \beta \text { when }} \\ \pi<2 \beta<\min ^{m}\left\{2 \pi, \tan \zeta_{1}+\zeta_{2}\right\}\end{array}\right.$

where $\zeta \in(-\infty, 0)$ satisfies $[\zeta / \tanh \zeta] \tan \zeta_{1}+\zeta_{2}=2 \beta$ when $\tan \zeta_{1}+\zeta_{2} \leqq 2 \beta<2 \pi$

For the class $\mathscr{L}_{1 / 2}$ we define $\mu\left(\beta ; \frac{1}{2}\right)=\nu\left(\beta ; \frac{1}{2}\right)=\pi / 2 \beta$.

A sketch of $\mu$ and $\nu$ as functions of $\alpha$ and $\beta$ is shown in Figure 1. Note that they are monotone decreasing functions of $\beta$ and that $\mu(\pi / 2 ; \alpha)=1$ for all $0<\alpha \leqq \frac{1}{2}$.

Next we define the periodic functions $C_{1}(\theta ; \beta ; \alpha)$ and $C_{2}(\theta ; \beta ; \alpha)$ parametrically as

$$
\begin{aligned}
C_{1}(\theta ; \beta ; \alpha) & =\frac{\cos \varphi\left\{1+\mu_{1} \cos \varphi\right\}^{(|\mu-1|-1) / 2}}{\left\{1+\mu_{2} \cos \varphi\right\}^{(|\mu-1|+1) / 2}} \\
\theta & =\frac{(4 \alpha(1-\alpha))^{1 / 2}}{\mu} \int_{0}^{\varphi} \frac{d \xi}{\left(1+\mu_{1} \cos \xi\right)\left(1+\mu_{2} \cos \xi\right)}
\end{aligned}
$$




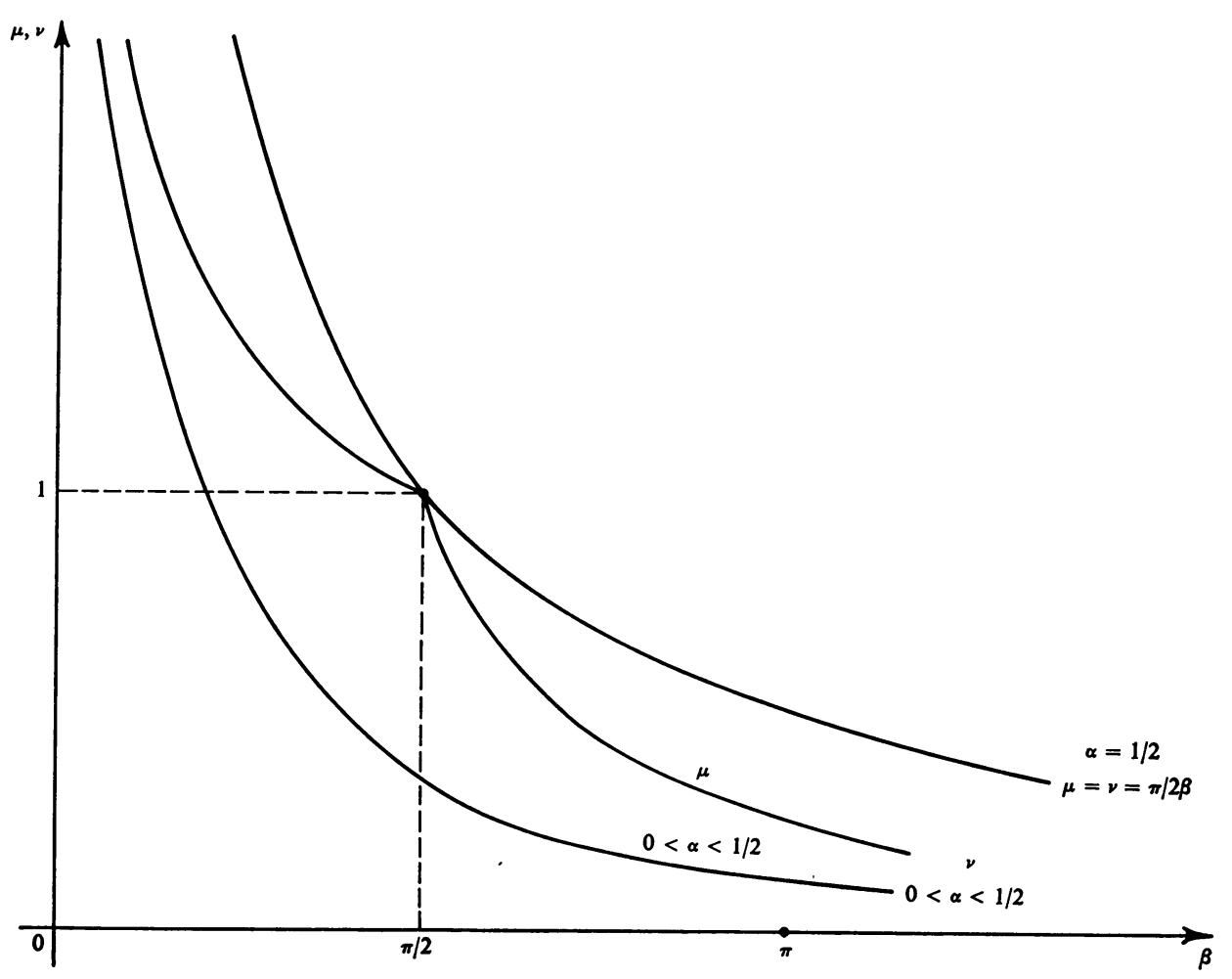

FIGURE 1

where $\mu_{1}=(|\mu-1|-1)(1-2 \alpha) / \mu$ and $\mu_{2}=(|\mu-1|+1)(1-2 \alpha) / \mu$ and

$$
\begin{aligned}
C_{2}(\theta ; \beta ; \alpha) & =\frac{\cos \varphi\left\{1+\nu_{1} \cos \varphi\right\}^{\nu / 2}}{\left\{1+\nu_{2} \cos \varphi\right\}^{(v+2) / 2}} \\
\theta & =\frac{(4 \alpha(1-\alpha))^{1 / 2}}{\nu} \int_{0}^{\varphi} \frac{d \xi}{\left(1+\nu_{1} \cos \xi\right)\left(1+\nu_{2} \cos \xi\right)}
\end{aligned}
$$

where $\nu_{1}=(1-2 \alpha)$ and $\nu_{2}=(\nu+2)(1-2 \alpha) / \nu$.

Our comparison function for the sector $S(\beta)$ is then given either by

$$
V_{1}(x, y)=r^{\mu} C_{1}(\theta ; \beta ; \alpha)
$$

or

$$
V_{2}(x, y)=r^{-v} C_{2}(\theta ; \beta ; \alpha)
$$

both of which are positive in the sector and vanish on its sides. Again they have been obtained in [16] as solutions of the maximizing equation relative to the class $\mathscr{L}_{\alpha}$ and have the property that

$$
L V(x, y) \leqq 0 \quad \forall(x, y) \in S(\beta)
$$


for every operator $L \in \mathscr{L}_{\alpha}$. Moreover for each of them there exists an operator $L^{\prime} \in \mathscr{L}_{\alpha}$ such that $L^{\prime} V=0$ in $S(\beta)$.

Since $\mu$ and $\nu$ are both positive we may refer to $V_{1}$ as an antibarrier at infinity and $V_{2}$ as an antibarrier at the origin, in the terminology of [15].

Note that for $\alpha=\frac{1}{2}$ these functions reduce to the harmonic functions

$$
V_{1}(x, y)=r^{\pi / 2 \beta} \cos \pi \theta / 2 \beta \quad V_{2}(x, y)=r^{-\pi / 2 \beta} \cos \pi \theta / 2 \beta .
$$

Furthermore for the case of a half plane $(\beta=\pi / 2)$ we have $\mu(\beta ; \alpha) \equiv 1$ and it is easily shown that the comparison function $V_{1}$ reduces to

$$
V_{1}(x, y)=\frac{r \cos \theta}{(4 \alpha(1-\alpha))^{1 / 2}}=\frac{x}{(4 \alpha(1-\alpha))^{1 / 2}} .
$$

3. The Phragmèn-Lindelöf theorem in a half strip. We shall first prove a preliminary version of the theorem and then proceed to sharpen it.

THEOREM 3.1. Let $D$ be an unbounded, open, connected set contained within a half strip of width $2 d$ in the plane. Let $B$ denote the finite boundary of $D$. Let $u(x, y)$ be a real valued function, twice differentiable in $D$, such that $L u \leqq 0$ in $D$ for some $L \in \mathscr{L}_{\alpha}$ and $\lim _{(x, y) \in D ;(x, y) \rightarrow B} \inf u(x, y) \geqq 0$. Suppose in addition that for some $\varepsilon>0$ we have $u^{-}(x, y)=o\left\{e^{(\lambda-\varepsilon) s}\right\}$ for $(x, y) \in D$ as $s \rightarrow \infty$, where $s$ denotes distance measured along the axis of the half strip and $\lambda=\lambda(d ; \alpha)$. Then $u \geqq 0$ in $D$.

Proof. Since the class of operators $\mathscr{L}_{\alpha}$ is invariant under rotation and translation of coordinates there is no loss in generality in assuming that $D$ is contained in the half strip $H(d)=\{(x, y):-d<x<d, y>0\}$.

From formula (2.1) it is clear that there exists a $d^{*}>d$ such that $\lambda^{*} \equiv \lambda\left(d^{*} ; \alpha\right)$ $=\lambda(d ; \alpha)-\varepsilon$. We consider now the comparison function $V(x, y)=e^{\lambda^{*} y} C\left(\lambda^{*} x ; \alpha\right)$.

From our previous remarks in $\S 2$ we have $L V \leqq 0$ in $D$. Moreover since $C\left(\lambda^{*} x ; \alpha\right)$ is strictly positive for all $(x, y) \in D \cup B$ the growth condition $u^{-}=o\left\{e^{\lambda^{*} u}\right\}$ as $y \rightarrow \infty$ may be written equivalently in the form

$$
u^{-}(x, y)=o\{V(x, y)\} \text { for all }(x, y) \in D \text { as } y \rightarrow \infty .
$$

The result now follows from Theorem 2.1 , taking $\Gamma_{1}=B$ and $\Gamma=\{\infty\}$.

Our purpose now is to improve this theorem by removing the $\varepsilon$ and to show that such a result is the best possible $\left({ }^{2}\right)$. We shall need the following

LEMma 3.2. Suppose that all hypotheses of Theorem 3.1 hold with the exception of the condition $m \equiv \lim _{(x, y) \in D ;(x, y) \rightarrow B}$ inf $u(x, y) \geqq 0$. Then $u \geqq m$ in $D$.

Proof. If $m=-\infty$ there is nothing to prove. If $-\infty<m<\infty$ we define $w(x, y)$ $=u(x, y)-m$ in $D$. Then we have $L w=L u \leqq 0$ in $D$ while $\lim _{(x, y) \in D ;(x, y) \rightarrow B}$ inf $w(x, y)$

$\left(^{2}\right)$ The possibility of improving Theorem 3.1 in this manner was first suggested to the author by Professor Keith Miller of the University of California, Berkeley. 
$\geqq 0$. Moreover the growth condition $w^{-}=o\left\{e^{(\lambda-\varepsilon) s}\right\}$ holds as $s \rightarrow \infty$. From Theorem 3.1 we conclude that $w \geqq 0$ in $D$.

From this result it follows readily that the case $m=+\infty$ cannot occur.

THEOREM 3.3. Let D be an unbounded, open, connected subset of the plane which, for suitably large values of $r\left(=\left(x^{2}+y^{2}\right)^{1 / 2}\right)$, is contained within a half strip of width $2 d$. Let $B$ denote the finite boundary of $D$. Let $u(x, y)$ be a real valued function, twice differentiable in $D$, such that $L u \leqq 0$ in $D$ for some $L \in \mathscr{L}_{\alpha}$ and

$$
\lim _{(x, y) \in D ;(x, y) \rightarrow B} \inf u(x, y) \geqq 0 .
$$

Suppose in addition that the growth condition $u^{-}(x, y)=o\left\{e^{\lambda s}\right\}$ holds for $(x, y) \in D$ as $s \rightarrow \infty$, where $s$ denotes distance measured along the axis of the half strip and $\lambda=\lambda(d ; \alpha)$. Then $u \geqq 0$ in $D$. Moreover this result is the best possible for the class of operators $\mathscr{L}_{\alpha}$.

Proof. Again by rotation and translation of coordinates we may assume that the half strip of the theorem is $H(d)=\{(x, y):-d<x<d, y>0\}$.

Let us first assume that $D$ is entirely contained in $H(d)$. From the growth condition on $u(x, y)$ we note that given any $\delta>0$ there exists a $\bar{y}(\delta)$ such that $u(0, y)$ $+\delta e^{\lambda y} \geqq 0$ for all points $(0, y) \in D$ with $y \geqq \bar{y}$.

We define $w(x, y)=u(x, y)+\delta e^{\lambda y} C(\lambda x ; \alpha) / C(0 ; \alpha)$ for $(x, y) \in D$. The proof will be accomplished if we can show that $w \geqq 0$ in $D$ for then fixing $(x, y) \in D$ and letting $\delta \rightarrow 0$ we obtain the desired result.

Let us define $H_{1}=\{(x, y):-d<x<0, y>\bar{y}\}, D_{1}=D \cap H_{1}$ and assume that $D_{1} \neq \varnothing$. Then we have $L w \leqq 0$ in $D_{1}$. Moreover since $w^{-}(x, y)=o\left\{e^{\lambda(d ; \alpha) y}\right\}$ for $(x, y) \in D$ as $y \rightarrow \infty$ and $\lambda(d ; \alpha)<\lambda(d / 2 ; \alpha)$ we have $w^{-}(x, y)=o\left\{e^{[\lambda(d / 2 ; \alpha)-\varepsilon] y}\right\}$ for $(x, y) \in D_{1}$ as $y \rightarrow \infty$ for sufficiently small $\varepsilon>0$.

Let $B_{1}$ denote the finite boundary of $D_{1}$. At any point $P$ of $B_{1}$ which also belongs to $B$ we have $\lim _{(x, y) \in D ;(x, y) \rightarrow P}$ inf $w(x, y) \geqq 0$. At those points of $B_{1}$ which are interior to $D$ and on the line $x=0$ we have $w(0, y)=u(0, y)+\delta e^{\lambda y} \geqq 0$. The remainder of $B_{1}$ consists of those points interior to $D$ which are on the line $-d<x<0, y=\bar{y}$.

Applying Lemma 3.2 (or the maximum principle in the case that $D_{1}$ is bounded and not empty) we obtain the following possibilities:

(1) $D_{1}=\varnothing$;

(2) $w \geqq 0$ in $D_{1}$;

(3) $w$ restricted to $\bar{D}_{1}$ attains its negative minimum at some point interior to $D$ and on the line $-d<x<0, y=\bar{y}$.

In the same way if we define $H_{2}=\{(x, y): 0<x<d, y>\bar{y}\}$ and $D_{2}=D \cap H_{2}$ we have the possibilities:

(1) $D_{2}=\varnothing$;

(2) $w \geqq 0$ in $D_{2}$;

(3) $w$ restricted to $\overline{D_{2}}$ attains its negative minimum at some point interior to $D$ and on the line $0<x<d, y=\bar{y}$. 
Finally applying the maximum principle to the remaining bounded set $\Omega=$ $D \cap\{(x, y): 0<y<\bar{y}\}$ we obtain the possibilities:

(1) $\Omega=\varnothing$;

(2) $w \geqq 0$ in $\Omega$;

(3) $w$ restricted to $\bar{\Omega}$ attains its negative minimum at some point interior to $D$ and on the line $-d<x<d, y=\bar{y}$.

Combining these results we conclude that either $w \geqq 0$ in $D$ or else $w$ attains its negative minimum at some point interior to $D$ and on the line $-d<x<d, y=\bar{y}$. We may further assert that the second possibility cannot occur for then the strong maximum principle would imply that $w$ is identically constant in $D$ which contradicts the assumption that $u^{-}(x, y)=o\left\{e^{\lambda y}\right\}$ for $(x, y) \in D$ as $y \rightarrow \infty$. Thus $w \geqq 0$ in $D$ and the proof is complete for this first case.

More generally a bounded open portion $D^{*}$ of the domain $D$ will extend beyond the base of the half strip $H(d)$. In this case let us define $m=\inf _{(x, 0) \in D ;-d<x<d} u(x, 0)$.

If $m \geqq 0$ we may apply the part of the theorem already proven to conclude that $u \geqq 0$ in the portion of $D$ which is contained in $H(d)$ and the maximum principle to conclude that $u \geqq 0$ in $D^{*}$. Combining these results we have $u \geqq 0$ in $D$.

If $m<0$ then $m$ must be attained at some point interior to $D$ and on the line segment $y=0,-d<x<d$. Defining $w=u-m$ and applying the part of the theorem already proven we find that $w \geqq 0$ in the portion of $D$ which is contained in $H(d)$. Again by the maximum principle we have $u \geqq m$ in $D^{*}$. Thus $u \geqq m$ in $D$ while $m$ is attained at some point interior to $D$ and on the line segment $y=0,-d<x<d$. The strong maximum principle then implies that $u \equiv m$ in $D$ which contradicts the hypothesis $\lim _{(x, y) \in D ;(x, y) \rightarrow B}$ inf $u(x, y) \geqq 0$. This concludes the proof.

Our result is the best possible for the class $\mathscr{L}_{\alpha}$ in the sense that the growth condition $o\{\}$ cannot be replaced by the weaker condition $O\{\}$. To show this consider the function $u(x, y)=1-e^{\lambda y} C(\lambda x ; \alpha) / C(0 ; \alpha)$ in the half strip $H(d)$ and note that on the finite boundary of $H(d)$ we have $u \geqq 0$ and, by our previous remarks in $\S 2$, there exists an $L^{\prime} \in \mathscr{L}_{\alpha}$ such that $L^{\prime} u=0$ in $H(d)$. While the growth condition $u^{-}=O\left\{e^{\lambda y}\right\}$ holds as $y \rightarrow \infty$ the condition of the theorem, $u^{-}=o\left\{e^{\lambda y}\right\}$ as $y \rightarrow \infty$, is violated. Since the conclusion of Theorem 3.3 does not hold for this function we conclude that the Phragmèn-Lindelöf growth index $\lambda(d ; \alpha)$ is the best possible for the class $\mathscr{L}_{\alpha}$.

The use of Theorem 3.3 (instead of Theorem 3.1) in Lemma 3.2 yields an analogous improvement of the extended minimum principle stated there.

Corollary (Extended Minimum Principle). Suppose that all hypotheses of Theorem 3.3 hold with the exception of the condition $m \equiv \lim _{(x, y) \in D ;(x, y) \rightarrow B} \inf u(x, y)$ $\geqq 0$. Then $u \geqq m$ in $D$.

Finally, applying the above corollary to the functions $u(x, y)$ and $-u(x, y)$, we obtain the following uniqueness result. 
COROllary (UNIQUeness). Let $D$ be an unbounded, open, connected subset of the plane which, for suitably large values of $r$, is contained in a half strip of width $2 d$. Let $B$ denote the finite boundary of $D$. Let $u(x, y)$ be a real valued function, twice differentiable in $D$ and continuous on $D \cup B$, such that $L u=0$ in $D$ for some $L \in \mathscr{L}_{\alpha}$ and $u=0$ on $B$. Suppose in addition that $u(x, y)=o\left\{e^{\lambda s}\right\}$ for $(x, y) \in D$ as $s \rightarrow \infty$, where $s$ denotes distance measured along the axis of the half strip and $\lambda=\lambda(d ; \alpha)$. Then $u \equiv 0$ in $D \cup B$.

REMARK. For simplicity we have considered only domains $D$ which, for suitably large values of $r$, are entirely contained within a single half strip; however the results may be extended easily to domains which, for suitably large values of $r$, are contained within a finite number, $N$, of half strips. In this case we would require a growth condition in each of the half strips (of widths $2 d_{1}, 2 d_{2}, \ldots, 2 d_{N}$ ) of the form $u^{-}=o\left\{e^{\lambda_{i} s}\right\}$ as $s \rightarrow \infty$ where $\lambda_{i}=\lambda\left(d_{i} ; \alpha\right)$ for $i=1, \ldots, N$.

4. The Phragmèn-Lindelöf theorem in a sector. Aside from the change in geometry from half strips to sectors and the use of the comparison functions (2.8) or (2.9) instead of (2.3) the proofs of the results to be given here are virtually identical with those in $\S 3$. For this reason we shall be content to merely state the theorems in their final forms.

THEOREM 4.1. Let $D$ be an unbounded, open, connected subset of the plane which, for suitably large values of $r\left(=\left(x^{2}+y^{2}\right)^{1 / 2}\right)$, is contained within a sector of half angle $\beta, 0<\beta<\pi$. Let $B$ denote the finite boundary of $D$. Let $u(x, y)$ be a real valued function, twice differentiable in $D$, such that $L u \leqq 0$ in $D$ for some $L \in \mathscr{L}_{\alpha}$ and $\lim _{(x, y) \in D:(x, y) \rightarrow B} \inf u(x, y) \geqq 0$. Suppose in addition that the growth condition $u^{-}(x, y)=o\left\{\rho^{\mu}\right\}$ holds for $(x, y) \in D$ as $\rho \rightarrow \infty$, where $\rho$ denotes distance measured from the vertex of the sector and $\mu=\mu(\beta ; \alpha)$. Then $u \geqq 0$ in $D$. Moreover this result is the best possible for the class of operators $\mathscr{L}_{\alpha}$.

The fact that the result is optimal for the class $\mathscr{L}_{\alpha}$ follows from the observation that the negative function $u(x, y)=-r^{\mu} C_{1}(\theta ; \beta ; \alpha)$ satisfies all conditions of the theorem in the sector $S(\beta)=\{(x, y): r>0,|\theta|<\beta<\pi\}$ except the growth restriction. In fact while the growth condition $u^{-}=O\left\{r^{\mu}\right\}$ holds as $r \rightarrow \infty$ the condition $u^{-}=o\left\{r^{\mu}\right\}$ is obviously violated.

Corollary (Extended Minimum Principle). Suppose that all hypotheses of Theorem 4.1 hold with the exception of the condition $m \equiv \lim _{(x, y) \in D ;(x, y) \rightarrow B} \inf u(x, y)$ $\geqq 0$. Then $u \geqq m$ in $D$.

Corollary (UNIQUeNESS). Let $D$ be an unbounded, open, connected subset of the plane which, for suitably large values of $r$, is contained within a sector of half angle $\beta$, $0<\beta<\pi$. Let $B$ denote the finite boundary of $D$. Let $u(x, y)$ be a real valued function, twice differentiable in $D$ and continuous on $D \cup B$, such that $L u=0$ in $D$ for some $L \in \mathscr{L}_{\alpha}$ and $u=0$ on $B$. Suppose in addition that $u(x, y)=o\left\{\rho^{\mu}\right\}$ for $(x, y) \in D$ as 
$\rho \rightarrow \infty$, where $\rho$ denotes distance measured from the vertex of the sector and $\mu=\mu(\beta ; \alpha)$. Then $u \equiv 0$ in $D \cup B$.

REMARK. The above results remain valid for a domain which, for suitably large values of $r$, is contained within a finite number of sectors of half angles $\beta_{1}, \ldots, \beta_{N}$ provided the appropriate growth condition $u^{-}=o\left\{\rho^{\mu_{i}}\right\}$ holds in each sector as $\rho \rightarrow \infty$, where $\mu_{i}=\mu\left(\beta_{i} ; \alpha\right)$ for $i=1, \ldots, N$.

REMARK. For the special case of a half plane sector $(\beta=\pi / 2)$ we have $\mu(\beta ; \alpha) \equiv 1$ for all $\alpha, 0<\alpha \leqq \frac{1}{2}$ and the result of Theorem 4.1 coincides with the well-known Phragmèn-Lindelöf theorem given by Gilbarg [1] and extended by Hopf [2].

THEOREM 4.2. Let $D$ be an open, connected subset of the plane which, in a neighbourhood of a finite boundary point $P$, is contained within a sector of half angle $\beta$, $0<\beta<\pi$ and vertex $P$. Let $B$ denote $\partial D-\{P\}$. Let $u(x, y)$ be a real valued function, twice differentiable in $D$, such that $L u \leqq 0$ in $D$ for some $L \in \mathscr{L}_{\alpha}$ and

$$
\lim _{(x, y) \in D:(x, y) \rightarrow B} \inf u(x, y) \geqq 0 .
$$

Suppose in addition that the growth condition $u^{-}(x, y)=o\left\{\rho^{-v}\right\}$ holds for $(x, y) \in D$ as $\rho \rightarrow 0$, where $\rho$ denotes distance measured from $P$ and $\nu=\nu(\beta ; \alpha)$. Then $u \geqq 0$ in $D$. Moreover this result is the best possible for the class of operators $\mathscr{L}_{\alpha}$.

The negative function $u(x, y)=-r^{-v} C_{2}(\theta ; \beta ; \alpha)$ in the sector

$$
S(\beta)=\{(x, y): r>0,|\theta|<\beta<\pi\}
$$

provides the example which shows that the Phragmèn-Lindelöf growth index $\nu(\beta ; \alpha)$ cannot be improved for the class $\mathscr{L}_{\alpha}$.

Corollary (Extended Minimum Principle). Suppose that all hypotheses of Theorem 4.2 hold with the exception of the condition $m \equiv \lim _{(x, y) \in D ;(x, y) \rightarrow B} \inf u(x, y)$ $\geqq 0$. Then $u \geqq m$ in $D$.

Corollary (UNIQUeness). Let $D$ be an open, connected subset of the plane which, in a neighbourhood of a finite boundary point $P$, is contained within a sector of half angle $\beta, 0<\beta<\pi$ and vertex $P$. Define $B=\partial D-\{P\}$. Let $u(x, y)$ be a real valued function, twice differentiable in $D$ and continuous on $D \cup B$, such that $L u=0$ in $D$ for some $L \in \mathscr{L}_{\alpha}$ and $u=0$ on $B$. Suppose in addition that $u(x, y)=o\left\{\rho^{-v}\right\}$ for $(x, y) \in D$ as $\rho \rightarrow 0$, where $\rho$ denotes distance measured from $P$ and $\nu=\nu(\beta ; \alpha)$. Then $u \equiv 0$ in $D \cup B$.

Remark. The results of Theorem 4.2 and its corollaries remain valid for a domain which, in a neighbourhood of each of a finite number of finite boundary points $P_{1}, \ldots, P_{N}$ is contained within a sector of half angle $\beta_{i}, 0<\beta_{i}<\pi$ and vertex $P_{i}$ for $i=1, \ldots, N$ provided the appropriate growth condition $u^{-}=o\left\{\rho^{-v_{i}}\right\}$ holds in each sector as $\rho \rightarrow 0$, where $\nu_{i}=\nu\left(\beta_{i} ; \alpha\right)$ for $i=1, \ldots, N$. A combination of Theorems 
4.1 and 4.2 may also be stated which would permit an a priori growth of solutions at both infinity and the vertex of a sector.

\section{REFERENCES}

1. D. Gilbarg, The Phragmèn-Lindelöf theorem for elliptic partial differential equations, J. Rational Mech. Anal. 1 (1952), 411-417.

2. E. Hopf, Remarks on the preceding paper by D. Gilbarg, J. Rational Mech. Anal. 1 (1952), 418-424.

3. A. Huber, A theorem of Phragmèn-Lindelöf type, Proc. Amer. Math. Soc. 4 (1953), 852-857.

4. J. Serrin, On the Phragmèn-Lindelöf principle for elliptic differential equations, J. Rational Mech. Anal. 3 (1954), 395-413.

5. A. Friedman, On two theorems of Phragmèn-Lindelöf for linear elliptic and parabolic differential equations of the second order, Pacific J. Math. 7 (1957), 1563-1575.

6. P. D. Lax, A Phragmèn-Lindelöf theorem in harmonic analysis and its application to some questions in the theory of elliptic equations, Comm. Pure Appl. Math. 10 (1957), 361-389.

7. S. Agmon and L. Nirenberg, Properties of solutions of ordinary differential equations in Banach space, Comm. Pure Appl. Math. 16 (1963), 121-239.

8. E. M. Landis, Some questions on the qualitative theory of elliptic equations of the second order, Uspehi Mat. Nauk 18 (1963), 3-62= Russian Math. Surveys 18 (1963), 1.

9. K. Habetha, Zum Phragmèn-Lindelöfschen Prinzip bei partiellen Differentialgleichungen, Arch. Math. 15 (1964), 324-331.

10. J. O. Herzog, Phragmèn-Lindelöf theorems for second order quasilinear elliptic partial differential equations, Proc. Amer. Math. Soc. 15 (1964), 721-728.

11. P. C. Fife, Growth and decay properties of solutions of second order elliptic equations, Ann. Scuola Norm. Sup. Pisa 20 (1966), 675-701.

12. J. K. Oddson, Phragmèn-Lindelöf and comparison theorems for elliptic-parabolic differential equations, Canad. J. Math. 19 (1967), 864-871.

13. - Phragmèn-Lindelöf and comparison theorems for elliptic equations with mixed boundary conditions, Arch. Rational Mech. Anal. 26 (1967), 316-334.

14. C. Pucci, Un problema variazionale per $i$ coefficienti di equazioni differenziali di tipo ellittico, Ann. Scuola Norm. Sup. Pisa 16 (1962), 159-172.

15. —- Operatori ellittici estremanti, Ann. Mat. Pura Appl. 72 (1966), 141-170.

16. J. K. Oddson, Some solutions of elliptic extremal equations in the plane, Matematiche (Catania) 23 (1968), 273-289.

17. K. Miller, Extremal barriers in cones with Phragmèn-Lindelöf theorems and other applications, (to appear).

UNIVERSITY OF CALIFORNia,

Riverside, CALIFORNIA 